TIPA. Travaux interdisciplinaires sur la parole et le langage

$31 \mid 2015$

L'impact du contact entre les langues

\title{
Les théories de l'accessibilité référentielle et la résolution des rapports anaphoriques ambigus au sein des textes classiques
}

The theories of referential accessibility and the resolution of ambiguous anaphoric relations in classical French

\section{Achraf Ben Arbia}

\section{OpenEdition \\ Journals}

Édition électronique

URL : http://journals.openedition.org/tipa/1477

DOI : 10.4000/tipa. 1477

ISSN : 2264-7082

Éditeur

Laboratoire Parole et Langage

Référence électronique

Achraf Ben Arbia, «Les théories de l'accessibilité référentielle et la résolution des rapports

anaphoriques ambigus au sein des textes classiques », TIPA. Travaux interdisciplinaires sur la parole et le langage [En ligne], 31 | 2015, mis en ligne le 22 décembre 2015, consulté le 26 mai 2020. URL:

http://journals.openedition.org/tipa/1477 ; DOI : https://doi.org/10.4000/tipa.1477

Ce document a été généré automatiquement le 26 mai 2020

\section{(c) (†) $\ominus$}

La revue TIPA. Travaux interdisciplinaires sur la parole et le langage est mise à disposition selon les termes de la licence Creative Commons Attribution - Pas d'Utilisation Commerciale - Pas de Modification 4.0 International 


\section{Les théories de l'accessibilité référentielle et la résolution des rapports anaphoriques ambigus au sein des textes classiques}

The theories of referential accessibility and the resolution of ambiguous anaphoric relations in classical French

Achraf Ben Arbia

\section{Introduction}

1 Dans cette contribution, la gestion des pronoms anaphoriques dans les textes classiques sera étudiée d'un point de vue cognitif, contrairement à ce que voudraient les grammairiens et les remarqueurs ${ }^{1}$ classiques. Ce choix méthodologique n'est pas sans importance. Il permet de dépasser les limites de l'approche textuelle sur laquelle se fonde le recrutement de l'antécédent en français classique. Ces limites sont dues à la capacité des expressions anaphoriques pronominales d'avoir un autre fonctionnement outre que le fonctionnement en tant qu'anaphoriques. Dans ce sens, leur interprétation référentielle n'est pas exclusivement textuelle. Autrement dit, il s'avère impossible, dans certaines situations discursives, de les rattacher à un antécédent facilement repérable. Dans ce même cadre d'analyse, Kleiber (1994), en parlant du pronom personnel il comme l'exemple prototypique de l'anaphore, signale que ce dernier peut avoir un autre fonctionnement, outre qu'anaphorique ${ }^{2}$. Il peut fonctionner également comme déictique. De ce fait, la localisation de l'antécédent du pronom personnel il n'est pas textuelle, mais situationnelle, liée à la situation d'énonciation.

2 Attention! ne t'approche pas. Il est dangereux ${ }^{3}$ (emploi de il sans antécédent prononcé par le père dans la situation où le fils s'approche trop près du chien). 
3 Dans cet énoncé, la localisation de l'antécédent du pronom personnel il est loin d'être contextuelle. Ce pronom acquiert dans ce cas un fonctionnement déictique. De ce fait, son interprétation dépend de la situation d'énonciation. C'est essentiellement pour cette raison, qui fait apparaître la disparité au niveau du fonctionnement des expressions référentielles, que Kleiber (1994a) prône le recours à une approche cognitive ou mémorielle lorsqu'il s'agit d'étudier l'anaphore. Cette approche est définie par Kleiber (1994a) comme un processus qui indique une référence à un référent déjà connu par l'interlocuteur; c'est-à-dire un référent présent ou déjà manifeste dans la mémoire immédiate $[. . .]^{4}$. Le recours à cette approche cognitive peut également être justifié selon Berrendonner (1983) par la capacité du pronom anaphorique d'avoir comme antécédent aussi bien un événement extra-linguistique qu'un segment de discours antérieur ${ }^{5}$. Comme nous l'avons déjà signalé, la question de la référence pronominale sera abordée sous un angle cognitif qui tiendra compte du rôle de la saillance des référents, mis en relation avec la structure informationnelle, à désigner un référent accessible référentiellement. La notion d'accessibilité, en tant que phénomène cognitif, désigne la capacité des expressions référentielles à fournir des informations sur le degré d'accessibilité cognitive de la représentation du référent. Dans ce sens, attribuer à un référent un degré d'accessibilité (fort, moyen ou faible) fait intervenir un certain nombre de facteurs à la fois cognitifs et linguistiques. La théorie de l'accessibilité [...] a vu la lumière en réaction à l'approche localisante des expressions référentielles selon laquelle le type d'expression référentielle dépend largement du contexte dans lequel se situe son référent ${ }^{6}$. Cette théorie, fondée sur l'accessibilité des référents, vise à indiquer à l'interlocuteur, sur la base de dispositifs mis en œuvre par le locuteur, le degré d'accessibilité que présente le référent. La théorie de l'accessibilité fait l'objet de plusieurs études qui sont pour la majorité complémentaires étant donné qu'elles aboutissent toutes au même résultat, celui d'assigner à une expression référentielle un degré d'accessibilité maximal par l'intersection de divers facteurs. Pour parvenir à classer les entités anaphoriques selon leur degré d'accessibilité, nous allons essayer de présenter dans ce qui va suivre trois approches fondamentales, à la fois syntaxiques et cognitives, établies respectivement par Ariel $(1988,1990,2001)$, par Keenan et Comrie $(1977,1979,1981)$ et par Chafe $(1987$, 1994) tout en mettant l'accent sur l'apport de chacune d'elles dans le processus d'interprétation des rapports anaphoriques ambigus dans les textes classiques.

\section{La théorie de l'accessibilité selon Ariel (1988, 1990, 2001)}

4 La théorie de l'accessibilité, telle qu'elle a été présentée par Ariel $(1988,1990,2001)^{7}$, est en rapport direct avec la théorie de cohérence dans la mesure où elle présente les marques du degré d'accessibilité des expressions référentielles au sein d'un texte. Elle dépasse ainsi le cadre strict de la phrase pour n'interpréter le degré d'accessibilité des référents qu'au niveau du texte. Ce degré d'accessibilité dépend en particulier de la distance entre l'expression référentielle et son antécédent. Autrement dit, plus la distance est réduite entre l'expression référentielle et l'antécédent, plus le degré d'accessibilité du référent est élevé. De ce fait, l'accessibilité référentielle joue un rôle déterminant dans le maintien de la cohérence textuelle étant donné qu'elle vise à rendre, en tenant compte de différents facteurs, un référent facilement accessible et présent dans la mémoire immédiate de l'interlocuteur, une fois que ce dernier tente de 
faire des prédictions sur le degré d'accessibilité que présente le référent au sein de l'énoncé. Attribuer à un référent un degré d'accessibilité (élevé, moyen ou faible) suggère le recours à différents facteurs complémentaires. Ariel $(1988,1990,2001)$ signale l'importance de quatre facteurs qui permettent à l'interlocuteur de juger le degré d'accessibilité des référents :

- Le premier facteur est relatif à la distance entre le référent et l'expression référentielle. Pour Ariel, plus celle-ci est proche du référent, plus ce dernier est accessible et présent à court terme dans la mémoire de l'interlocuteur ;

- Le deuxième facteur est lié au degré de saillance du référent. Plus celui-ci est saillant (se trouve déjà dans la mémoire immédiate de l'interlocuteur), plus il est accessible référentiellement ;

- Le troisième facteur est un facteur positionnel. Autrement dit, plus la distance entre l'expression anaphorique et son antécédent est grande, moins le référent est accessible. L'unité entre l'expression anaphorique et le référent est un facteur important permettant à l'interlocuteur de faire des prédictions sur le degré d'accessibilité d'un référent ;

- Le quatrième facteur concerne la compétition entre les référents. Plus un fragment de texte contient des référents potentiellement concurrents, moins le référent auquel renvoie l'expression anaphorique est accessible.

Tous ces facteurs développés par Ariel contribuent à déterminer le degré d'accessibilité d'un référent à un moment donné du discours. Toutefois, il convient de les étudier tous dans leur complémentarité, puisque focaliser l'attention sur un seul facteur semble insuffisant pour dégager le degré d'accessibilité d'une expression référentielle. Ainsi, l'interlocuteur peut-il accéder dans certaines situations au référent indéterminé référentiellement et à propos duquel il s'avère difficile d'appliquer certains calculs inférentiels sans recourir aux divers facteurs de l'accessibilité référentielle. Dans ce cas, le référent présentant un degré d'accessibilité élevé n'est plus le GN récemment introduit dans le discours, mais tout simplement le topique du discours. La notion de topique sera abordée dans le dernier chapitre de ce travail, notamment en rapport avec les phénomènes de continuité topicale/de changement du topique. Toutefois, il convient de préciser que le topique du discours peut être identifié sans recourir aux différents facteurs de la théorie d'accessibilité présentée par Ariel étant donné que ce dernier est défini par Grobet (2002) et Corburn (1992) comme a) le point de départ de l'énoncé, b) l'élément porteur du plus bas degré de dynamisme communicatif, c) l'information donnée ou d) ce dont parle l'énoncé. Ainsi, pour reconnaître le topique de l'énoncé, l'interlocuteur peut ne pas tenir compte des facteurs mis à sa disposition par la théorie de l'accessibilité étant donné que le topique possède déjà un degré d'accessibilité élevé. Cependant, l'application de la théorie d'accessibilité n'est pas sans importance. Ariel rattache cette importance au fait que le degré d'accessibilité s'explique par le degré d'ambiguïté de l'expression référentielle. Autrement dit, le degré d'accessibilité varie d'une expression référentielle à une autre. Un référent accessible référentiellement et présentant un degré d'accessibilité élevé par rapport à d'autres référents présents dans le même contexte serait le référent qui répond parfaitement aux différents facteurs de la théorie de l'accessibilité (distance, saillance, unité et compétition). Dans l'énoncé (1), le référent approprié de l'expression anaphorique pronominale il acquiert un degré d'accessibilité élevé par rapport au deuxième antécédent concurrent vu qu'il répond aux différents facteurs de la théorie de l'accessibilité référentielle : 
1) Si M. de Sévigné était ici, il vous rendrait grâce, comme moi, des offres que vous lui faites, mais notre ami Bussy vous pourra dire où il est depuis deux mois.

Contentez-vous donc de mes seules connaissances, et de la protestation que je vous fais de vous honorer plus que tous les hommes du monde. Il est impossible d'avoir eu l'honneur de vous voir sans avoir pour vous une estime tout extraordinaire, et puisque souvent nous avons pensé crever de rire ensemble, faites vos conclusions, et jugez vous-même que je suis avec passion, monsieur, votre très humble et obéissante servante. (Mme de Sévigné, Correspondance, t, 1, 1646-1675, 1675, p. 11, 1649)

Dans cet énoncé, les deux premières occurrences du pronom personnel de troisième personne il et lui sont coréférentielles, puisqu'elles réfèrent toutes les deux à l'antécédent M. de Sévigné. Cependant, la troisième occurrence du même pronom dans mais notre ami Bussy pourra vous dire où il est depuis deux mois est ambigu, puisque un nouveau référent concurrent, à savoir notre ami Bussy figure dans la même phrase. L'ambiguïté référentielle se justifie dans cet énoncé par la capacité du pronom personnel il à référer à l'un des deux antécédents potentiellement concurrents $M$. de Sévigné et notre ami Bussy. En tenant compte des apports de la théorie de l'accessibilité, telle qu'elle a été présentée par Ariel (1988), nous allons essayer, sur la base des différents facteurs (distance, unité, saillance et compétition), de localiser le bon référent contextuel de l'expression anaphorique pronominale ambiguë $i l$. Dans ce sens, ce référent sera au premier plan dans la représentation du discours du lecteur. Il est considéré, dans ce même cadre d'analyse, comme étant de forte accessibilité référentielle par rapport au deuxième référent concurrent et auquel tous les pronoms anaphoriques réfèrent. En appliquant les différents facteurs de l'accessibilité référentielle fournis par Ariel (1988), nous pouvons trancher que, parmi les deux antécédents concurrents $M$. de Sévigné et notre ami Bussy, c'est le premier référent qui constitue l'antécédent visé par le pronom personnel il étant donné qu'il répond aux différents facteurs qui permettent de lui conférer un degré d'accessibilité élevé. Le tableau mentionné ci-dessous résume l'application des facteurs de l'accessibilité référentielle sur les deux antécédents concurrents :

Enoncé 1

Tableau 1 : L'application des facteurs de l'accessibilité référentielle selon Ariel (1988, 1990, 2001)

\begin{tabular}{|l|l|l|}
\hline $\begin{array}{l}\text { Facteurs de l'accessibilité } \\
\text { référentielle selon Ariel } \\
(1988)\end{array}$ & $\begin{array}{l}\text { Antécédent potentiel 1 } \\
\text { M. de Sévigné }\end{array}$ & Antécédent potentiel 2 \\
\hline Distance & $\begin{array}{l}\text { Le pronom et l'antécédent } \\
\text { figurent dans la même phrase. }\end{array}$ & $\begin{array}{l}\text { Le pronom et l'antécédent } \\
\text { figurent dans la même phrase. }\end{array}$ \\
\hline Saillance & $\begin{array}{l}\text { [+ saillant }] \\
\text {-Position initiale dans la phrase. } \\
\text {-Repris par plusieurs pronoms } \\
\text { coréférentiels (il, lui). }\end{array}$ & $\begin{array}{l}\text { [- saillant }] \\
\text { phrase. } \\
\text {-Introduit récemment dans le } \\
\text { discours. }\end{array}$ \\
\hline
\end{tabular}




\begin{tabular}{|l|l|l|}
\hline Unité & $\begin{array}{l}\text {-Dans la même phrase que le } \\
\text { pronom (sujet d'une proposition } \\
\text { subordonnée). }\end{array}$ & $\begin{array}{l}\text {-Dans la même phrase que le } \\
\text { pronom (sujet d'une phrase } \\
\text { coordonnée à la première). }\end{array}$ \\
\hline Compétition & $\begin{array}{l}\text {-Thème de l'énoncé (présent dans } \\
\text { la mémoire discursive des } \\
\text { interlocuteurs). } \\
\text {-Plus focalisé }\end{array}$ & $\begin{array}{l}\text {-Antécédent concurrent } \\
\text { (récemment introduit dans le } \\
\text { discours). } \\
\text {-Moins focalisé }\end{array}$ \\
\hline $\begin{array}{l}\text { Degré d'accessibilité } \\
\text { référentielle }\end{array}$ & [+accessible] & [-accessible] \\
\hline
\end{tabular}

Comme le montre ce tableau, l'accessibilité du référent est influencée par tous ces facteurs. Ces derniers, réunis ensemble, confèrent à l'un des deux antécédents concurrents un degré d'accessibilité élevé. Dans ce sens, cet antécédent, ayant une forte accessibilité référentielle, constitue le référent auquel renvoie l'expression anaphorique pronominale ambiguë. Certes, la corrélation entre tous ces facteurs peut guider le lecteur à localiser l'antécédent d'une expression référentielle ambiguë, mais comme nous l'avons déjà montré, l'interlocuteur peut accéder dans certains cas au référent sans la nécessité d'appliquer tous ces facteurs, non parce que ce référent a été mentionné récemment, mais parce qu'il s'agit du topique du discours, présent dans la mémoire discursive des interlocuteurs. Dans cette perspective, il importe d'ajouter que notre recours à la théorie de l'accessibilité proposée par Ariel, en dépit des critiques qui lui sont adressées, constitue une tentative de désambiguïsation des énoncés classiques dans lesquels se bloque toute interprétation référentielle en termes de rapport anaphorique entre pronom/antécédent. A côté de la théorie de l'accessibilité figurent d'autres approches qui visent également à classifier les référents concurrents, présents pour la même occurrence pronominale, selon leur degré d'accessibilité référentielle. Ces approches sont conçues par plusieurs linguistes dont notamment, Keenan et Comrie $(1977,1979,1981)$, Lehmann $(1984,1986)$, etc. Chacune d'elles a pour objectif de mettre en avant les différents facteurs qui déterminent le degré d'accessibilité d'un référent à un moment donné du discours. Après avoir présenté la théorie de l'accessibilité selon Ariel (1988), nous allons à présent parcourir l'étude de Keenan et Comrie $(1977,1979,1981)$ de l'accessibilité référentielle des référents tout en essayant d'adopter ses apports pour la résolution des rapports anaphoriques ambigus dans les textes classiques.

\section{La théorie de l'accessibilité selon Keenan et Comrie $(1977,1979,1981)$}

9 Rappelons, avant d'aborder la théorie de l'accessibilité proposée par Keenan et Comrie (1977, 1979, 1981), que l'anaphore pronominale sera abordée comme une marque linguistique de la cohérence d'un discours ${ }^{8}$. Dans ce sens, les pronoms anaphoriques, en tant que moyens cohésifs assurant la continuité référentielle, sont des marqueurs privilégiés permettant l'accès à l'entité référentielle la plus focalisée. Néanmoins, et comme nous l'avons déjà démontré à maintes reprises, les pronoms anaphoriques peuvent faire l'objet de différentes orientations référentielles. Autrement dit, 
l'interprétation référentielle de ces derniers peut poser problème lors d'une situation référentielle ambiguë. Cette indétermination dans les rattachements référentiels ne fait qu'interrompre la continuité référentielle et informationnelle au sein de l'énoncé. C'est en particulier dans les textes classiques que la gestion des pronoms anaphoriques se heurte à ce genre d'indétermination référentielle. Cette incohérence référentielle est due essentiellement à la capacité des pronoms anaphoriques de désigner des référents potentiellement concurrents ayant souvent le même degré d'accessibilité référentielle. La récupération de l'antécédent approprié pour le terme de reprise, en termes de calculs inférentiels, est fondée sur un seul principe dont les limites ne permettent pas la localisation du bon référent. Il s'agit bien évidemment de l'approche textuelle dont l'application pousse, dans certains cas, le lecteur à localiser l'entité référentielle, visée par le pronom anaphorique. Le recours à cette approche peut également guider le lecteur vers le choix de l'entité référentielle la moins saillante et la moins focalisée, lorsque ce dernier rattache le pronom anaphorique au GN le plus proche sans aucun critère de sélection et en dépit de la présence d'une expression référentielle concurrente susceptible de constituer, elle aussi, le topique du discours. Dans l'énoncé (2), le recrutement de l'antécédent, visé par l'expression anaphorique pronominale elle, suggère différentes orientations référentielles :

2) Voici une pièce d'une construction assez extraordinaire : aussi est-ce la vingt et unième que j'ai fait voir sur le théâtre ; et après y avoir fait réciter quarante mille vers, il est bien malaisé de trouver quelque chose de nouveau, sans s'écarter un peu du grand chemin, et se mettre au hasard de s'égarer. La tendresse et les passions, qui doivent être l'âme des tragédies, n'ont aucune part en celle-ci : la grandeur de courage y règne seule, et regarde son malheur d'un œil si dédaigneux qu'il n'en saurait arracher une plainte. Elle y est combattue par la politique, et n'oppose à ses artifices qu'une prudence généreuse, qui marche à visage découvert, qui prévoit le péril sans s'émouvoir, et ne veut point d'autre appui que celui de sa vertu, et de l'amour qu'elle imprime dans les cœurs de tous les peuples (Corneille, Nicomède, Au lecteur, 1651, p. 501).

La résolution de cet énoncé, en termes de rattachement référentiel de l'antécédent à l'expression anaphorique pronominale, se heurte à différentes interprétations référentielles. Le pronom anaphorique elle est source d'ambiguïté anaphorique étant donné qu'il est susceptible de reprendre l'un des deux référents concurrents la grandeur de courage et une plainte, appartenant tous les deux au même fragment textuel. Notons par ailleurs que nombreux sont les facteurs qui interviennent lors de l'attribution des référents aux expressions anaphoriques pronominales. Ces facteurs sont d'ordre syntaxique, sémantique et pragmatique. Toutefois, l'interprétation référentielle du rapport anaphorique sur la base des deux facteurs syntaxique et sémantique peut échouer quand il y a plusieurs antécédents syntaxiquement ou/et sémantiquement compatibles avec marqueur anaphorique ambigu. Pour l'aspect pragmatique de l'anaphore, la présence de plusieurs antécédents saillants et présents dans la mémoire discursive des interlocuteurs peut empêcher toute résolution anaphorique univoque. Reste enfin à voir le rôle de l'expression anaphorique pronominale dans le maintien de la continuité topicale au sein du discours. Au niveau local du paragraphe, c'est le degré de saillance du référent qui assure son maintien en tant que topique du discours. Pour Lambrecht (1994), certaines expressions anaphoriques pronominales s'emploient 
exclusivement pour assurer une continuité topicale au sein de l'énoncé. Autrement dit, ces expressions marquent la continuité topicale en désignant un référent qui est topique dans la phrase ou dans la proposition. C'est le cas des pronoms personnels de la troisième personne qui sont dotés de la capacité à reprendre un référent d'une part saillant et de l'autre considéré comme topique de l'énoncé. Reprenons ainsi l'exemple (16) pour vérifier la capacité du pronom personnel elle à maintenir la continuité topicale au sein du paragraphe. Dans cet énoncé, le morphème anaphorique elle, ambigu, admet deux statuts référentiels opposés. Le premier est lié à sont rôle en tant que marqueur de continuité topicale, référant ainsi au topique de l'énoncé. La deuxième acception de ce pronom lui confère un autre rôle lié à sa capacité d'indiquer un changement au niveau du topique du discours. Dans ce sens, la présence d'un autre référent concurrent n'est autre qu'une contrainte vis-à-vis de l'étude de la continuité topicale censée être assurée par les expressions anaphoriques pronominales. Face à cette indétermination référentielle et à la possibilité d'un changement au niveau du topique, un classement des référents concurrents selon leur degré d'accessibilité référentielle s'avère d'une grande importance. Ce degré d'accessibilité peut être mesuré par la valeur syntaxique qu'occupent les antécédents concurrents au sein de l'énoncé. Dans ce cadre, la théorie de l'accessibilité référentielle proposée par Keenan et Comrie $^{9}(1977,1979)$ permet, dans une certaine mesure, de résoudre les rapports anaphoriques ambigus en conférant au référent désigné par l'anaphorique un degré de continuité topicale au sein de l'énoncé. Pour ces deux grammairiens, les syntagmes nominaux présentent différents degrés d'accessibilité référentielle selon leurs positions syntaxiques dans la phrase. Autrement dit, le processus de référenciation devrait tenir compte de la valeur syntaxique que possède le référent au sein de l'énoncé. Les référents sont ainsi localisés par des calculs inférentiels qui tiennent compte de la fonction syntaxique des groupes nominaux concurrents. Selon cet angle d'analyse, l'échelle d'accessibilité référentielle telle qu'elle a été établie par Keenan et Comrie $(1977,1979)$ attribue aux groupes nominaux occupant la fonction sujet un degré d'accessibilité référentielle élevé par rapport aux groupes nominaux occupant une fonction syntaxique régie par le verbe. Selon cette optique, l'antécédent ayant la fonction sujet au sein de l'énoncé jouit d'une forte accessibilité référentielle par rapport à un autre antécédent concurrent ayant la fonction de complément d'objet direct. Le complément d'objet direct est plus accessible que le complément d'objet indirect. Le complément d'objet indirect serait également plus accessible que le complément circonstanciel. Cette classification des référents selon leurs fonctions syntaxiques permet de lever l'ambiguïté référentielle et de rattacher le pronom anaphorique à l'antécédent qui jouit d'une forte accessibilité référentielle par rapport aux autres référents concurrents présents également dans le même énoncé. Dans les deux énoncés (3) et (4), la localisation du référent approprié pour chacune des deux expressions anaphoriques pronominales en et qui se fonde sur la théorie de l'accessibilité référentielle proposée par Keenan et Comrie $(1977,1979)$ :

3) La proposition ne plut pas ; mais on ne put la refuser, et parce qu'elle était dans l'ordre, et parce qu'elle faisait, en quelque façon, juger que je voulais au moins retenir mes bénéfices, puisque j'en voulais prendre soin (Retz, Mémoires, p. 59). 
4) Meillaincour, écuyer de mon frère, qui me servait de second, et qui avait été blessé dans le petit ventre et désarmé, et le chevalier du Plessis, second de Praslin, nous vinrent séparer (Retz, Mémoires, p. 61).

11 Dans ces deux énoncés, l'attribution d'un référent à chacune des deux expressions anaphoriques pronominales en et qui pose problème. Ceci est dû à la présence d'autres référents saillants dans le même énoncé, ce qui rend le rattachement référentiel du terme de reprise à son antécédent plus difficile à établir. Néanmoins, en nous basant sur la hiérarchie de l'accessibilité (HA) proposée par Keenan et Comrie $(1977,1979)$, nous pouvons classer les référents selon la valeur syntaxique qu'ils occupent au sein de l'énoncé en référents ayant une forte accessibilité référentielle et en référents de faible accessibilité référentielle. Le premier énoncé (3) met ainsi en concurrence deux référents, l'un en fonction de sujet et l'autre en fonction de complément d'objet direct. Ainsi, l'antécédent la proposition, en position sujet, est-elle plus accessible que le deuxième GN concurrent mes bénéfices, en fonction de complément d'objet direct. Le pronom anaphorique en réfère dans ce cas à l'antécédent jouissant d'une forte accessibilité référentielle et constituant le thème de l'énoncé la proposition. Dans le deuxième énoncé (4), les deux occurrences du pronom relatif qui sont susceptibles de reprendre l'un des deux antécédents concurrents Meillaincour (écuyer) ou mon frère. Le premier antécédent Meillaincour (écuyer), sujet de l'énoncé, est plus saillant que le deuxième GN concurrent mon frère, ayant pour fonction complément du nom Meillancour. Le référent, présentant un degré d'accessibilité référentielle plus élevé, serait alors le sujet Meillaincour qui constitue le thème de l'énoncé et non l'antécédent le plus proche mon frère.

12 L'échelle de l'accessibilité référentielle des référents établie par Keenan et Comrie (1977, 1979), selon laquelle la classification des référents concurrents se fait en tenant compte de leurs fonctions syntaxiques dans l'énoncé, permet assez souvent, comme nous l'avons déjà montré, de résoudre certains rapports anaphoriques ambigus. L'application de la théorie d'accessibilité de Keenan et Comrie, visant à expliquer les préférences des rattachements référentiels d'un pronom anaphorique à son antécédent, permet également, à côté d'autres théories, de lever les ambiguïtés référentielles générées par une gestion libre des expressions anaphoriques pronominales en français classique. Cette théorie de l'accessibilité référentielle permet au lecteur moderne des textes classiques d'effectuer les calculs référentiels nécessaires et de comprendre le rôle des expressions anaphoriques pronominales dans le maintien d'une part de la continuité informationnelle et de l'autre de la continuité référentielle.

13 Les tableaux ci-dessous résument les préférences des rattachements référentiels appliquées dans les énoncés (2), (3) et (4) où tous les rapports anaphoriques sont ambigus

Tableau 2 : L'application des facteurs de l'accessibilité référentielle selon Keenan et Comrie (1977, 1979, 1981)

\begin{tabular}{|l|l|l|}
\hline $\begin{array}{l}\text { Facteurs de l'accessibilité } \\
\text { référentielle selon Keenan et } \\
\text { Comrie (1977) }\end{array}$ & $\begin{array}{l}\text { Antécédent potentiel 1 } \\
\text { La grandeur de courage }\end{array}$ & Unécédent potentiel 2 \\
\hline
\end{tabular}




\begin{tabular}{|c|c|c|}
\hline Distance & $\begin{array}{l}\text {-Le pronom et l'antécédent } \\
\text { appartiennent à deux segments } \\
\text { textuels différents. }\end{array}$ & $\begin{array}{l}\text {-Le pronom et l'antécédent } \\
\text { appartiennent à deux segments } \\
\text { textuels différents. }\end{array}$ \\
\hline Saillance & $\begin{array}{l}\text { [+saillant] } \\
\text {-Position initiale dans la phrase } \\
\text { (thème de l'énoncé). }\end{array}$ & $\begin{array}{l}\text { [-saillant] } \\
\text {-Position non initiale dans } \\
\text { l'énoncé. } \\
\text {-Récemment introduit dans le } \\
\text { discours. }\end{array}$ \\
\hline $\begin{array}{l}\text { Fonction syntaxique au sein } \\
\text { de l'énoncé }\end{array}$ & -Sujet de l'énoncé. & $\begin{array}{l}\text {-Fonction régie par le verbe } \\
\text { (COD). }\end{array}$ \\
\hline $\begin{array}{l}\text { Degré } \quad d \text { accessibilité } \\
\text { référentielle }\end{array}$ & $\begin{array}{l}\text {-Forte accessibilité référentielle. } \\
\text { [+accessible] }\end{array}$ & $\begin{array}{l}\text {-Faible } \\
\text { référentielle. } \\
\text { [-accessible] }\end{array}$ \\
\hline
\end{tabular}

Enoncé 3

Tableau 3 : L'application des facteurs de l'accessibilité référentielle selon Keenan et Comrie (1977, $1979,1981)$

\begin{tabular}{|c|c|c|}
\hline $\begin{array}{l}\text { Facteurs de l'accessibilité } \\
\text { référentielle selon Keenan et } \\
\text { Comrie (1977) }\end{array}$ & $\begin{array}{l}\text { Antécédent potentiel } 1 \\
\text { La proposition }\end{array}$ & $\begin{array}{l}\text { Antécédent potentiel } 2 \\
\text { Mes bénéfices }\end{array}$ \\
\hline Distance & $\begin{array}{l}\text {-Le pronom et l'antécédent } \\
\text { font partie du même segment } \\
\text { textuel. }\end{array}$ & $\begin{array}{l}\text {-Le pronom et l'antécédent } \\
\text { font partie du même segment } \\
\text { textuel. }\end{array}$ \\
\hline Saillance & \begin{tabular}{|l} 
[+saillant $]$ \\
-Position initiale dans \\
l'énoncé. \\
-Thème de l'énoncé.
\end{tabular} & $\begin{array}{l}\text { [-saillant] } \\
\text {-Position non initiale dans } \\
\text { l'énoncé. } \\
\text {-GN récemment introduit au } \\
\text { sein du discours. }\end{array}$ \\
\hline $\begin{array}{l}\text { Fonction syntaxique au sein de } \\
\text { l'énoncé }\end{array}$ & -Sujet de l'énoncé. & $\begin{array}{l}\text {-Fonction régie par le verbe } \\
\text { (COD). }\end{array}$ \\
\hline $\begin{array}{l}\text { Degré } \\
\text { référentielle }\end{array}$ & $\begin{array}{l}\text {-Forte accessibilité } \\
\text { référentielle. } \\
\text { [+accessible }]\end{array}$ & $\begin{array}{l}\text {-Faible accessibilité } \\
\text { référentielle. } \\
\text { [-accessible] }\end{array}$ \\
\hline
\end{tabular}

Enoncé 4 
Tableau 4 : L'application des facteurs de l'accessibilité référentielle selon Keenan et Comrie (1977, $1979,1981)$

\begin{tabular}{|c|c|c|}
\hline $\begin{array}{l}\text { Facteurs de l'accessibilité } \\
\text { référentielle selon Keenan et } \\
\text { Comrie (1977) }\end{array}$ & $\begin{array}{l}\text { Antécédent potentiel } 1 \\
\text { Meillaincour }\end{array}$ & $\begin{array}{l}\text { Antécédent potentiel } 2 \\
\text { Mon frère }\end{array}$ \\
\hline Distance & $\begin{array}{l}\text {-Le pronom et l'antécédent } \\
\text { font partie du même segment } \\
\text { textuel. }\end{array}$ & $\begin{array}{l}\text {-Le pronom et l'antécédent font } \\
\text { partie du même segment textuel. }\end{array}$ \\
\hline Saillance & $\begin{array}{l}\text { [+saillant] } \\
\text {-Nom propre: thème de } \\
\text { l'énoncé. } \\
\text {-Position initiale dans la } \\
\text { phrase. }\end{array}$ & $\begin{array}{l}\text { [-saillant] } \\
\text {-Structure : } N \text { de } N . \\
\text {-Position non initiale dans la } \\
\text { phrase. } \\
\text {-GN récemment introduit dans le } \\
\text { discours. }\end{array}$ \\
\hline $\begin{array}{l}\text { Fonction syntaxique au sein } \\
\text { de l'énoncé }\end{array}$ & -Sujet de l'énoncé. & $\begin{array}{l}\text {-Complément du nom Meillancour } \\
(\mathrm{N} \text { de } N) \text {. }\end{array}$ \\
\hline $\begin{array}{l}\text { Degré } \\
\text { référentielle }\end{array}$ & $\begin{array}{l}\text {-Forte accessibilité } \\
\text { référentielle. } \\
\text { [+accessible] }\end{array}$ & $\begin{array}{l}\text {-Faible accessibilité référentielle. } \\
\text { [-accessible] }\end{array}$ \\
\hline
\end{tabular}

\section{La théorie de l'accessibilité selon Chafe (1987, 1994)}

Chafe $(1987,1994)^{10}$ établit une classification des référents selon l'opposition entre référent donné et référent nouveau. Pour ce linguiste, le degré d'accessibilité des référents varie selon leur degré de saillance et selon leur statut cognitif au sein de l'énoncé. Dans cette même perspective, Chafe établit une opposition entre référent donné qu'il considère comme une entité référentielle introduite explicitement dans l'univers du discours et doté d'un degré de saillance bien particulier et référent nouveau qui ne possède aucune de ses propriétés et qui est considéré comme inactif dans la mémoire discursive des interlocuteurs. En établissant cette classification, Chafe (1994) propose trois facteurs primordiaux lors de l'identification des référents. Ces facteurs rendent compte également du degré d'activation ${ }^{11}$ des référents dans la mémoire discursive des interlocuteurs

- l'identification du référent suppose des connaissances partagées par le locuteur et l'interlocuteur : référent supposé partagé entre locuteur et interlocuteur ;

- le référent, activé dans la mémoire discursive des interlocuteurs, doit être désigné par une expression référentielle qui rendra facile son identification ;

- le référent doit être contextuellement saillant.

Pour Chafe (1994), il existe une relation étroite entre le statut cognitif du référent et son interprétation en tant que topique. Autrement dit, pour qu'un référent soit interprété en tant que topique du discours, il faut qu'il soit suffisamment accessible et 
activé dans la mémoire discursive des interlocuteurs. De ce fait, les référents qui sont dotés d'un degré d'accessibilité élevé tout en étant actifs au sein du discours sont considérés comme donnés. Par contre, les référents récemment introduits dans le discours, inactifs et qui deviennent au fur et à mesure actifs sont considérés comme nouveaux. La classification des référents selon cette opposition entre référent donné et référent nouveau telle qu'elle a été établie par Chafe (1994) peut être synthétisée de la manière suivante :

Tableau 5 : L'activation des référents selon Chafe (1994)

\begin{tabular}{|c|c|c|c|}
\hline $\begin{array}{l}\text { Coût } \\
\text { d } \\
\text { 'activation }\end{array}$ & $\begin{array}{l}\text { Statut du référent dans } \\
\text { l'unité d'intonation } 1\end{array}$ & $\begin{array}{l}\text { Statut du référent dans } \\
\text { l'unité d'intonation } 2\end{array}$ & $\begin{array}{l}\text { Type } \\
\text { d'activation }\end{array}$ \\
\hline \multirow[t]{2}{*}{-} & Référent actif 䦪 & Référent actif & $=$ référent donné \\
\hline & Référent semi-actif 撕 & Référent actif & $\begin{array}{l}=\quad \text { référent } \\
\text { accessible }\end{array}$ \\
\hline+ & Référent inactif 韵 & Référent actif & $\begin{array}{l}=\quad \text { référent } \\
\text { nouveau }\end{array}$ \\
\hline
\end{tabular}

Selon cette schématisation du degré d'activation des référents, un référent donné est un référent actif, saillant et présent dans la mémoire discursive des interlocuteurs. Un référent nouveau est un référent qui acquiert un degré d'activation et d'accessibilité référentielle une fois que sa représentation au sein du discours devient d'une part permanente et de l'autre, liée aux connaissances partagées par les interlocuteurs. Sur la capacité des pronoms démonstratifs à désigner un référent qui n'est pas encore accessible dans la mémoire discursive des interlocuteurs, Kleiber (1991b : 155) signale que :

«[...] le fait d'introduire un référent nouveau contraint par avance l'élément déjà connu que représente lui à ne plus pourvoir être qu'une classe de $N$ ou d'humains. Voici pourquoi. Comme celui-ci/là introduit dans ce cas de figure un référent nouveau saisi dans la classe déjà manifeste des référents à laquelle renvoie lui, cette classe ne peut rassembler une classe de référents eux-mêmes déjà connus. Dit autrement, les lui ne peuvent être des référents particuliers déjà introduits, sinon le référent amené à la saillance par la saisie démonstrative ne peut plus lui-même être un référent nouveau, puisqu'il est extrait de classe déjà saillante ${ }^{12}$

Cette opposition entre référent nouveau et référent connu suggère différents degrés d'activation référentielle des référents présents au sein du même énoncé. Dans ce sens, l'application de la théorie d'accessibilité référentielle établie par Chafe (1994), sur des énoncés classiques, vise davantage à classer les référents selon leur statut cognitif (leur degré d'activation) qu'à décrire le fonctionnement des expressions référentielles. Dans l'énoncé (5), cette dichotomie entre référent donné et référent nouveau permet d'accéder au référent visé par l'expression anaphorique pronominale et de classer les référents selon leur degré d'activation référentielle : 
5) La tyrannie consiste au désir de domination universel et hors de son ordre. Diverses chambres de forts, de beaux, de bons esprits, de pieux, dont chacun règne chez soi, non ailleurs. Et quelques fois ils se rencontrent et le fort et le beau se battent sottement à qui sera le maître l'un de l'autre, car leur maîtresse est de divers genre. Ils ne s'entendent pas. Et leur faute est de vouloir régner partout. Rien ne le peut, non pas même la force : elle ne fait rien au royaume des savants, elle n'est maîtresse que des actions extérieures. (Pascal, Pensées, 1662, p. 85, III, Misère, 54)

La résolution des anaphores pronominales et l'attribution de relations entre les entités $\mathrm{du}$ discours ou les segments textuels retenus assurent la progression informationnelle et permettent la compréhension du sens global du texte. Or, il arrive que l'identification des relations entre les entités référentielles soit difficile, voire impossible. Autrement dit, l'assignation d'un pronom de reprise à son propre antécédent ouvre le champ à différentes interprétations référentielles souvent contradictoires. Ces constructions anaphoriques ambigües sont assez répandues dans les textes classiques. De ce fait, l'évolution de la règle grammaticale en français classique et la multiplication des cas de restrictions vis-à-vis de certains faits de langue ne font que rendre plus difficile la localisation précise du référent lors d'une concurrence référentielle entre plusieurs antécédents présents pour le même marqueur anaphorique. Face à ces différentes situations d'indétermination référentielle parcourues dans les textes classiques, il s'avère important de prendre en compte certaines approches facilitant l'accessibilité des référents. Le recours à ces approches modernes permet dans la majorité des cas de résoudre les ambiguïtés référentielles engendrées lors d'une mauvaise gestion des pronoms anaphoriques au sein des textes classiques. La cohérence textuelle nécessite ainsi le recours à certains facteurs d'ordre pragmatique pour mettre en relation les morphèmes anaphoriques avec leurs propres antécédents. Notons également dans ce même cadre d'analyse que, selon la hiérarchie d'accessibilité référentielle présentée par Chafe (1994), le degré d'activation des référents dans la mémoire discursive des interlocuteurs varie selon le statut des GN antécédents au sein de l'énoncé : référent donné/référent nouveau. Dans ce sens, un référent nouveau serait moins actif qu'un référent donné, présent déjà dans la mémoire discursive des interlocuteurs. Ainsi, dans l'exemple (5) les deux référents concurrents la tyrannie et la force acquièrent, de par leur position dans l'énoncé, un degré d'accessibilité référentielle différent. C'est à travers leur statut cognitif, en termes de connaissances partagées, que l'interlocuteur parvient à rattacher les deux occurrences ambigües du pronom personnel elle à l'antécédent le plus saillant la tyrannie. Le degré d'activation d'un référent repose selon Chafe (1994) sur trois facteurs. Ainsi, un référent facilement identifiable est, tout d'abord, présupposé connu par le locuteur et l'interlocuteur. Ensuite, il doit être également désigné par une expression référentielle qui rend facile son identification. Enfin, il doit acquérir un degré de saillance contextuelle élevé en situation de concurrence référentielle. De ce fait, l'antécédent la tyrannie, constituant le thème de l'énoncé, est l'entité référentielle active et centrale dans la représentation mentale des interlocuteurs. Ce statut cognitif lui confère un degré de saillance contextuelle élevé par opposition au deuxième GN concurrent la force. Tous ces facteurs permettent aux interlocuteurs d'interpréter la référence pronominale dans le cas où la structure du discours ne fournit pas une base 
informationnelle suffisante pour ces opérations. La classification des référents concurrents selon leur degré d'activation (Chafe, 1994) peut être schématisée de la manière suivante :

Tableau 6 : L'application des Facteurs de l'accessibilité référentielle selon Chafe $(1987,1994)$

\begin{tabular}{|c|c|c|}
\hline $\begin{array}{l}\text { Facteurs de l'accessibilité } \\
\text { référentielle selon Chafe (1994) }\end{array}$ & $\begin{array}{l}\text { Antécédent potentiel } 1 \\
\text { La tyrannie }\end{array}$ & $\begin{array}{l}\text { Antécédent potentiel } 2 \\
\text { La force }\end{array}$ \\
\hline $\begin{array}{l}\text { Degré de } \\
\text { contextuelle }\end{array}$ & $\begin{array}{l}\text { [+saillant] } \\
\text {-Thème de l'énoncé. } \\
\text {-Position initiale dans l'énoncé } \\
\text { (sujet). } \\
\text {-Référent saillant dans la mémoire } \\
\text { discursive des interlocuteurs. } \\
\rightarrow \text { Degré de saillance locale élevé. }\end{array}$ & $\begin{array}{l}\text { [-saillant] } \\
\text {-Position non initiale } \\
\text { dans l'énoncé. } \\
\text {-GN récemment introduit } \\
\text { dans le discours. } \\
\rightarrow \text { Degré de saillance } \\
\text { locale faible. }\end{array}$ \\
\hline Type d'activation & $\begin{array}{l}\text {-Référent donné. } \\
\text {-Référent actif. } \\
\text {-Référent accessible. }\end{array}$ & $\begin{array}{l}\text {-Référent nouveau. } \\
\text {-Référent inactif. } \\
\text {-Référent inaccessible. }\end{array}$ \\
\hline $\begin{array}{l}\text { Degré } \\
\text { référentielle }\end{array}$ & $\begin{array}{l}\text { [+activé] } \\
\text { Degré d'activation référentielle } \\
\text { élevé. }\end{array}$ & $\begin{array}{l}\text { [-activé }] \\
\text { Degré d'activation } \\
\text { référentielle faible. }\end{array}$ \\
\hline
\end{tabular}

D'après cette classification référentielle des référents selon leur degré d'activation, nous avons pu constater que chaque expression référentielle signale un statut cognitif particulier qui lui confère une saillance locale particulière dans l'énoncé. Celle-ci permet généralement de résoudre les situations anaphoriques ambigües en rattachant les morphèmes anaphoriques à leurs propres antécédents. Les différentes théories de l'accessibilité référentielle, passées en revue, permettent dans le cadre d'une concurrence référentielle entre plusieurs antécédents de classer ces derniers selon leur degré d'accessibilité en référents ayant une forte accessibilité référentielle, référents ayant une accessibilité référentielle moyenne et référents ayant une faible accessibilité référentielle.

\section{Conclusion}

Dans cet article, nous nous sommes intéressé à trois théories de l'accessibilité référentielle, développées respectivement par Ariel $(1988,1990,2001)$, Keenan et Comrie $(1977,1979,1981)$ et Chafe $(1987,1994)$. Chacune de ces théories permet une approche assez souple et dynamique des relations anaphoriques et semble être bien adaptée aux textes classiques. L'application de ces théories de l'accessibilité sur des énoncés classiques nous a permis d'établir un classement des entités référentielles concurrentes en nous fondant sur leur degré d'accessibilité référentielle. Ainsi, ces dernières sont réparties en référents ayant une forte accessibilité référentielle et référents ayant une faible accessibilité référentielle. L'application de chacune de ces 
trois théories tient compte d'un ensemble de facteurs, indispensables lors de la classification des référents concurrents selon leur degré d'accessibilité référentielle. Dans ce même cadre, il convient de signaler que le recours à ces approches modernes n'est pas sans importance. S'appuyer uniquement sur une approche strictement positionnelle, selon laquelle l'antécédent d'une expression anaphorique pronominale est le GN le plus proche en dépit de la présence d'autres référents référentiellement saillants, ne fait que multiplier les ambiguïtés référentielles au lieu de les résoudre. L'application de ces trois approches modernes a permis, comme nous l'avons démontré, de restreindre les ambiguïtés référentielles en guidant le lecteur actuel des textes classiques au bon référent visé par le pronom anaphorique.

\section{BIBLIOGRAPHIE}

Académie française, $(1719,1720)$, Remarques sur le « Quinte-Curce » de Vaugelas, édition critique par Wendy Ayres-Bennett et Philippe Caron, Presses de l'école supérieure, «Etudes et documents en histoire de la langue française », 1997.

Achard-Bayle, G., (1996), Référence, identité, changement : la désignation des référents évolutifs. Etudes de cas : les récits de métamorphoses, Thèse de doctorat, Nancy II.

Adam, J-M., (1999), Linguistique textuelle. Des genres du discours aux textes. Paris : Nathan.

Adam, J-M., Grize, J-B., Bouacha, M-A., (2004), Texte et discours : catégories pour l'analyse, Editions Universitaires de Dijon.

Aissani, A., (2006), L'anaphore résomptive dans le texte écrit en langue française par l'étudiant algérien, Thèse de doctorat, Université d'Alger.

Anscombre, J-C., (1990), Thème, espace discursif et référence événementielle, Fonctionnalisme et Pragmatique à propos de la notion de thème, Milano, Italie : Edizioni unicopli.

Apothéloz, D., (1995), Rôle et fonctionnement de l'anaphore dans la dynamique textuelle, Genève : Droz. Ariel, M., (1988), Referring and accessibility, Journal of Linguistics 24, p. 65-87.

Ariel, M., (1990), Accessing Noun-Phrase antecedents. London/New York: Routledge.

Ariel, M., (2001), Accessibility Theory: An Overview. In T. Sanders, J. Schilperood et W. Spooren (eds) Text representation: Linguistic and psycholinguistic aspects. Amsterdam/Philadelphia: John Benjamins, p. 29-87.

Chafe, W., (1987), Cognitive constraints on information flow. In R.S. Tomlin (ed.) Coherence and grounding in discourse. Outcome of a Symposium, Eugene, Oregon, June 1984. Amsterdam/Philadelphia: John Benjamins, p. 21-51.

Charolles, M., (1978), Introduction au problème de la cohérence des textes, Langue française 38, p. 7-41.

Charolles, M., (1988), Les études sur la cohérence, la cohésion et la connexité textuelles depuis la fin des années 1960, Modèles linguistiques 10/2, p. 45-66. 
Charolles, M., (1994), Cohésion, cohérence et pertinence du discours, Travaux de linguistique 29, p. $125-151$.

De Mulder, W., Co Vet et Vetters, C., (2001), Anaphores pronominales et nominales. Etudes pragmasémantique, Amsterdam, New York: Rodopi B.V

De Weck, G., (1991), La cohésion dans les textes d'enfants. Etude du développement des processus anaphoriques, Neuchâtel : Delachaux et Niestlé.

Delbecque, N., (2002), Linguistique cognitive, comprendre comment fonctionne le langage, De BoeckDuculot.

Demol, A., (2010), Les anaphoriques celui-ci et il :étude des facteurs qui déterminent leur choix, Université Gent : Academiejaar 2006-2007.

Dupuy-Parant, E., (2006), La continuité référentielle en moyen français : règles syntactico-sémantiques, Thèse de doctorat, soutenue le 15 septembre 2006, Université de Maine, Faculté des Lettres, Langues et Sciences Humaines.

Fournier, N., (1998), Grammaire du français classique, Paris : Belin.

Frantext, Base de données accessible en ligne.

Givón, T., (1983), Topic Continuity in Discourse: Quantitative Cross-language Studies, John Benjamins Publishing Company, Amsterdam, p. 12/ Code, Mind and Context. Chicago: Chicago University Press.

Givón, T., (1992), The grammar of referential coherence as mental processing instructions, Linguistics, 30, 1, p. 5-55.

Grobet, A., (2002), L'identification des topiques dans les dialogues, Bruxelles : De-Boeck-Duculot.

Grosz, B., (1977), The representation and use of focus in dialogue understanding, Technical Report 151, SRI International, 333 Ravenswood Ave, Menlo Park, Ca. 94025.

Guéron, J. (1979), Relations de coréférence dans la phrase et dans le discours, Langue française, 44, p. 42-79.

Kail, M., Léveillé, M., (1997), Compréhension de la coréférence des pronoms personnels chez l'enfant et l'adulte, L'année psychologique, 77, 1, p. 97-94.

Keenan, E., (1976), Towards a universal definition of "subject". In Ch. Li (ed.) Subject and topic. New York: Academic Press, p. 303-333.

Keenan, E., et Comrie, B., (1977), Noun phrase accessibility and Universal grammar, Linguistic Inquiry, 8, p. 63-99.

Keenan, E., et Comrie, B., (1979), Data of the noun phrase accessibility hierarchy, Language 55, p. 333-351.

Kintsch, W., et Van Dijk, T.-A., (1983), Strategies of discourse comprehension, New York: Academic Press.

Kleiber, G., (1989), Reprise(s). Recueil d'études sur les processus anaphoriques, Publication du Groupe Anaphore et Deixis, $n^{\circ} 1$, Strasbourg : Université des Sciences Humaines.

Kleiber, G., (1994), Anaphores et pronoms, Champs linguistiques, Louvain-la-Neuve : Duculot.

Trouilleux, F., (2001), Identification des reprises et interprétation automatique des expressions pronominales dans les textes en français, Thèse de doctorat, Université de Blaise Pascal, ClermontFerrand. 
Veland, R., (1996), Les marqueurs référentiels celui-ci et celui-là. Structure interne et déploiement dans le discours direct littéraire, Genève-Paris : Droz.

\section{NOTES}

1. Lors du rattachement d'un pronom anaphorique à son propre antécédent les grammairiens et les remarqueurs classiques, notamment Vaugelas, se basent sur une approche strictement textuelle selon laquelle le bon référent est le GN le plus proche du pronom de reprise. Dans cet article, la gestion des pronoms anaphoriques au sein des textes classiques sera abordée sous un angle cognitif qui mettra au premier plan les différents facteurs de l'accessibilité référentielle, en l'occurrence le degré d'activation des référents concurrents dans la mémoire discursive des interlocuteurs.

2. Sur cette même question, voir aussi Anne Zribi-Hertz (1992).

3. Kleiber, 1994a, Discours et stéréotypie : le contexte peut-il remettre d'aplomb une anaphore associative mal formée? L'Anaphore associative, Recherches linguistiques, XIX, C. Schnedecker, M. Charolles, G. Kleiber et J. David (éds). Paris : Klincksieck, p. 24.

4. Idem, p. 25.

5. Berrendonner, A., 1983, Cours critique de grammaire générative. Lyon : Presses Universitaires de Lyon ; Fribourg : Editions Universitaires, p. 225-226.

6. Annemie Demol, 2010, Les pronoms anaphoriques il et celui-ci, Duculot : Bruxelles, p. 128.

7. Ariel, M., 1988, Referring and accessibility, Journal of Linguistics 24, p. 65-87.

-Ariel, M., 1990, Accessing Noun-Phrase antecedents. London/New York: Routledge.

-Ariel, M., 2001, Accessibility Theory: An Overview. In T. Sanders, J. Schilperood et W. Spooren

(eds) Text representation: Linguistic and psycholinguistic aspects. Amesterdam/Philadelphia: John Benjamins, p. 29-87.

8. Reboul, A., 1997, (In) cohérence et anaphore : mythes et réalités, in de Mulder, W., TasmowskiDe Ryck, L. et Vetters, C. (éds) Relations anaphoriques et incohérence, Amsterdam: Rodopi,

p. 297-314.

9. Keenan, E., et Comrie, B., 1977, Noun phrase accessibility and Universal grammar, Linguistic Inquiry, 8, p. 63-99.

-Keenan, E., et Comrie, B., 1979, Data of the noun phrase accessibility hierarchy, Language 55, p. 333-351.

-Comrie, B., 1981, Language universals and linguistic typology: Syntax and morphology. Chicago: University of Chicago Press.

10. Chafe, W., 1987, Cognitive constraints on information flow. In R.S. Tomlin (ed.) Coherence and grounding in discourse. Outcome of a Symposium, Eugene, Oregon, June 1984. Amsterdam/Philadelphia: John Benjamins, p. 21-51.

-Chafe, W., 1994, Discourse, consciousness and time: the flow and displacement of conscious experience in speaking and writing. Chicago et London: The University of Chicago Press.

11. Sur la question du degré d'activation des référents, voir Lambrecht (1988, p. 144) : « du point de vue de l'activation, un référent discursif est activé s'il constitue à un moment donné le centre d'attention des participants au discours ». Cette définition du référent actif rejoint celle établie par (Gundel et al. 2000) : « une fois qu'une entité a revêtu le statut cognitif d'identifiable, elle le demeure tout au long du discours, mais elle peut aussi avoir un statut plus élevé dans la hiérarchie ».

12. Kleiber, G., 1991b, Prototype et prototypes : encore une affaire de famille, Danièle Dubois (dir.) Sémantique et Cognition : Catégorie, prototypes, typicalité : 103-130. Paris : Éditions du CNRS. p. 155. 


\section{RÉSUMÉS}

Dans cet article, au regard de l'approche textuelle et de son insuffisance lors de la résolution des rapports anaphoriques ambigus au sein des textes classiques, nous allons essayer d'appliquer d'autres approches modernes, à la fois syntaxiques et cognitives, qui s'avèreront appropriées lors du rattachement référentiel de l'expression anaphorique pronominale à son propre antécédent. Autrement dit, il sera question de passer en revue trois approches fondamentales, présentées respectivement par Ariel $(1988,1990,2001)$, Keenan et Comrie $(1977,1979,1981)$ et Chafe $(1987$, 1994), et de les appliquer à des énoncés référentiellement ambigus. Ces approches permettront l'éviction des ambiguïtés référentielles, dans le cadre d'une concurrence référentielle entre plusieurs antécédents pour le même marqueur anaphorique, en rattachant le terme de reprise à son propre antécédent. L'application de ces approches modernes permet d'assurer la continuité référentielle et informationnelle au sein des textes de la période classique tout en permettant leur intelligibilité.

The work on the textual organization (Apotheloz, 1995; Grobet, 2000; Combettes, 1986) have demonstrated the great importance that played the management of pronouns anaphoric in structuring textual. However, some attachments repositories are far away to comply with such a requirement and have the more often an influence of a side, on the organization of informational text and of the other, on its thematic progression. Let us note, from this same point of view, as the problems relating to the resolution of the pronominal anaphora, as regards the attachment repository of the pronoun anaphoric blizzard to the antecedent, are one of the most salient features of the classical language. The grammarians of this period work, essentially, on the eviction of equivocal order repository. The latter are likely to generate different interpretations often of the same report anaphoric blizzard. However, despite the established rules throughout the XVIIth century, aimed at restricting the use of free pronouns anaphoric, the management of these terms of resumption is the more often the source of referential ambiguities. These referential ambiguities constitute a side, one of the major obstacles to the reading of classic texts and on the other, are sources of textual inconsistency. In this sense, some reports anaphoric remain difficult to interpret because several history are presented as potential candidates for the same anaphoric pronoun. To resolve this type of equivocation, the conventional grammarians erected as the sole criterion for selection, the principle of proximity. This principle is to reattach the pronoun anaphoric to the closest referent in the left context. However, this criterion has proved, in some cases, unreliable in the measure or the referents competitors, present in the same statement, can, also, claim the role of antecedent while introducing a degree of referential accessibility high. On the basis of these different findings, we will try to look at the operation of the repository anaphoric pronouns from examples attested learned of various classical works. Our objective is also to demonstrate that the referential interpretation of these reports anaphoric within classical texts poses problems for the modern reader. In this same vein, we will try, first of all, to put the emphasis on the problems relating to the location of the referent of a pronoun anaphoric blizzard in a classical French and then, we will propose, based on different theoretical approaches presented respectively by (Keenan and Comrie (1977, 1979, 1981), Ariel $(1988,1990,2001)$, Chafe $(1987,1994))$, a classification of referents competitors according to their degree of referential accessibility. This referential classification will allow the resolution of reports anaphoric during a referential ambiguity. The application of the various factors of the referential accessibility on reports anaphoric ambiguous has proved of great importance to the extent or their application referentially indeterminate situations guide, generally, to the proper reference. Our results seem to confirm that the noun anaphora is not considered solely as a 
phenomenon dependent on its context and as a problem to be solved, but rather as a process of cognitive management of the dynamic discursive. Because of this, the management of pronouns anaphoric in the framework of a discursive situation fact appeal to different interpretative process to the time cognitive and syntactic. In this same perspective, the application of theories of the accessibility, presented respectively by Ariel (1988, 1990, 2001), Keenan and Comrie (1977, $1979,1981)$ and Chafe $(1987,1994)$, has allowed, in the framework of a referential competition, to classify referents according to their degree of referential accessibility. Several factors are involved, as well, in the process of benchmarking, and allow a hand to resolve the ambiguity in referential linking the pronoun anaphoric blizzard to the antecedent adequate and on the other to establish a prioritization at the level of the degree of accessibility of referents placed in competition. These are classes in referents having a strong referential accessibility, referents enjoying a referential accessibility average and referents having a low referential accessibility. This referential classification duct in the majority of cases to the allocation of a unique reference restricting, as well, the preferences of attachment repository.

\section{INDEX}

Mots-clés : anaphore pronominale, ambiguïté référentielle, accessibilité référentielle, saillance, théories de l'accessibilité référentielle

Keywords : pronominal anaphora, referential ambiguity, referential accessibility, salience, theories of referential accessibility

\section{AUTEUR}

\section{ACHRAF BEN ARBIA}

Faculté des Lettres et des Sciences Humaines de Kairouan, Laboratoire de recherche : Langues, Discours et Cultures (ISSH de Jendouba, Tunisie)

Enseignant-chercheur. Docteur en linguistique française. Domaine de recherche : les procédés de reprise (l'anaphore) ; la gestion des pronoms anaphoriques dans deux états de langue ; la langue du XVIIème siècle et la langue moderne achraf_benarbia@yahoo.fr 